\title{
The impact of having both cancer and diabetes on patient-reported outcomes: a systematic review and directions for future research
}

\author{
Pauline A. J. Vissers ${ }^{1,2}$ • Louise Falzon ${ }^{3}$ - Lonneke V. van de Poll-Franse ${ }^{1,2}$. \\ Frans Pouwer $^{1} \cdot$ Melissa S. Y. Thong ${ }^{1,2}$
}

Received: 16 June 2015 / Accepted: 6 September 2015 /Published online: 1 October 2015

(C) The Author(s) 2015. This article is published with open access at Springerlink.com

\begin{abstract}
Purpose This systematic review aims to summarize the current literature regarding potential effects of having both cancer and diabetes on patient-reported outcomes (PROs) and to provide directions for future research.

Methods MEDLINE, The Cochrane Library, CINAHL, and PsycINFO were searched from inception to January 2015. All English peer-reviewed studies that included patients with both cancer and diabetes and assessed PROs were included. All included studies were independently assessed on methodological quality by two investigators.

Results Of the 3553 identified studies, 10 studies were included and all were considered of high ( $40 \%)$ or adequate $(60 \%)$ methodological quality. Eight of the 10 studies focused on health-related quality of life (HRQoL), functioning, or symptoms and 2 studies assessed diabetes self-management. Overall, HRQoL and functioning was lower, and symptoms were higher among patients with both cancer and diabetes as compared to having cancer or diabetes alone. Furthermore, one
\end{abstract}

Electronic supplementary material The online version of this article (doi:10.1007/s11764-015-0486-3) contains supplementary material, which is available to authorized users.

Pauline A. J. Vissers

p.a.j.vissers@tilburguniversity.edu

1 CoRPS - Center of Research on Psychology in Somatic diseases, Department of Medical and Clinical Psychology, Tilburg University, P.O. Box 90153, 5000 LE Tilburg, The Netherlands

2 Department of Research, Netherlands Comprehensive Cancer Organisation, Eindhoven, The Netherlands

3 Center for Behavioral Cardiovascular Health, Columbia University Medical Center, New York, NY, USA study reported that diabetes self-management was impaired after chemotherapy.

Conclusions Having both cancer and diabetes resulted in worse PROs compared to having either one of the diseases, however, the considerable heterogeneity of the included studies hampered strong conclusions. Future studies are needed as this research area is largely neglected. As the majority of the included studies focused on HRQoL, future research should address the impact of both diseases on other PROs such as depression, patient empowerment and self-management. Implications for Cancer Survivor Having both cancer and diabetes might result in worse PROs, however, more research is needed as current evidence is scarce.

Keywords Cancer · Diabetes · Patient-reported outcomes · Systematic review $\cdot$ Health-related quality of life

\section{Introduction}

Due to the increased aging of the population, early detection, and better treatment of diseases, the number of cancer survivors is increasing [1]. As a result, more and more cancer survivors live with other chronic diseases of which diabetes is one of the most prevalent [2]. The prevalence of concurrent diabetes among cancer patients depends on cancer type, gender, and age at diagnosis and varies from $8 \%$ among prostate cancer patients to approximately $26 \%$ among pancreas cancer patients aged 65 years or older [2]. This high prevalence of diabetes among cancer patients results in worse outcomes and increases the burden on health systems worldwide.

The link between cancer and diabetes is extensively studied in recent literature and is mainly focused on the impact of diabetes on cancer incidence and mortality. Recent metaanalyses show that diabetes is strongly associated with the 
development of pancreatic (OR $=1.82,95 \%$ CI: $1.66-1.89)$ [3], liver ( $\mathrm{OR}=2.50,95 \%$ CI: $1.80-3.50)$ [4], and endometrial cancer $(\mathrm{RR}=2.10,95 \% \mathrm{CI}: 1.75-2.53)$ [5]. Moderate, positive associations have been reported for diabetes and breast $(\mathrm{RR}=1.20,95 \% \mathrm{CI}: 1.12-1.28)$ [6], colorectal $(\mathrm{RR}=1.26$, $95 \%$ CI: $1.05-1.50)$ [7], and bladder ( $R R=1.24,95 \% \mathrm{CI}$ : 1.08-1.42) [8] cancer incidence, while diabetes has been associated with a decreased incidence of prostate cancer $(\mathrm{RR}=$ 0.84, 95 \% CI: 0.76-0.93) [9]. Furthermore, previous research shows that having diabetes is associated with a $30-40 \%$ increased mortality risk among cancer patients, which was mainly apparent among breast, endometrial, and colorectal cancer patients $[10,11]$.

As the group of patients with both cancer and diabetes is growing, patients' experience of living with both diseases is becoming more important. However, this research area is largely neglected. Patient-reported outcome (PRO) assessments such as health-related quality of life (HRQoL), functioning, and symptoms are needed as it is plausible that patients with multiple chronic diseases experience more problems. This knowledge is essential to improve clinical practice and care for this growing group of patients.

A significant number of cancer survivors consistently report lower physical functioning, sexual functioning, and more symptoms of distress and fatigue $[12,13]$. Similarly, diabetes patients are more likely to suffer from depression [14], report a lower quality of life [15], and lower sexual functioning [16]. As both cancer and diabetes patients report deteriorated PROs compared to people without the disease, we hypothesize that having both chronic diseases will result in even more deteriorated PROs. The aim of this systematic literature review is to summarize the current knowledge on the impact of having both cancer and diabetes on PROs. In addition, as we expect that this research area will be largely neglected, we also aim to provide directions for future research.

\section{Methods}

\section{Search strategy}

LF conducted the systematic literature search on August 2013 and updated the search on January 2015. The following databases were included: MEDLINE, The Cochrane Library, CINAHL, and PsycINFO. Subject headings and freetext terms for diabetes (i.e., diabet* OR diabetes mellitus) were combined with search terms for cancer (i.e., cancer* OR neoplasm* OR oncolog*). As PROs cover a wide range of different aspects, we did not include any search terms for PROs to avoid missing relevant papers. The full search strategy is shown in Online Resource 1. After the search was conducted, the cited references of the selected studies were searched using Web of Science and their references lists checked; in addition,
PubMed related articles were used for the two most recent included studies to identify studies that were not found with the initial literature search.

\section{Selection criteria}

All retrieved studies (including abstracts of unpublished studies) were screened and studies that met the following four selection criteria were included: (1) the study is focused on patients with both cancer and diabetes, (2) PRO is primary or secondary outcome measure of the study, (3) is published in a peer-reviewed journal, and (4) is published in English. Studies that assessed the effects of several chronic or comorbid diseases, including diabetes, among cancer patients on PROs were not included as the studies should have a primary focus on both cancer and diabetes. Similarly, studies that aimed to address comorbid or chronic diseases, including cancer, among diabetes patients were excluded.

\section{Quality assessment}

Each selected study was independently scored on methodological quality by 2 reviewers (PV and MT) based on a set of 14 quality criteria (Table 1 ). These quality criteria were based on established criteria lists used in previous studies [17, 18]. Disagreements between the reviewers on the quality criteria were resolved during a consensus meeting. All studies received 1 point for each of the 14 quality criteria that was met. If a criterion was not met or described insufficiently, 0 point was assigned. Thus, each study can obtain a maximum score of 14 points. Studies that scored $75 \%$ or more of the maximum attainable score (i.e., $\geq 11$ points) were considered as "high quality study," studies scoring between 50-75\% (i.e., 7-10 points) were considered of "adequate quality," while those scoring $<50 \%$ (i.e., $\leq 6$ points) were considered of "low quality." These criteria were arbitrarily chosen and based on previous research [17].

\section{Results}

\section{Description of the included studies}

The initial broad search strategy on cancer and diabetes that did not include a term for "PROs" yielded 3553 hits, and after the removal of duplicates and the application of selection criteria, a total of 10 studies were included in this study, of which 2 were based on the same data [19, 20] (Fig. 1). Eight of the included studies had a sample size of at least 590 participants, while 2 studies, based on the same data, included 43 patients [19, 20] (Table 2). The number of patients with both cancer and diabetes was rather low; 5 studies included less than 100 patients with both diseases [19-23]. Moreover, only 
Table 1 List of criteria for assessing the methodological quality of studies on patient-reported outcomes among patients with cancer and diabetes

Positive if with respect to

Patient-reported outcomes

1. Examining PROs was a primary objective of the study

2. A validated questionnaire to measure PROs was used

Study population

3. The patient sampling process is described

4. A (healthy) normative sample is included for comparison

5. Patients with both cancer and diabetes are compared to either patients with only cancer or only diabetes on at least two sociodemographic variables

6. A description is included of at least two clinical variables regarding cancer diagnosis

(e.g., cancer stage, treatment, time since cancer diagnosis)

7. A description is included of at least two clinical variables regarding diabetes diagnosis or severity

(e.g., $\mathrm{HbA}_{1 \mathrm{c}}$ levels, treatment, time since diabetes diagnosis)

8. Inclusion and/or exclusion criteria are described

9. Participation rates for patient groups are described and these are $>75 \%$

10. Information is given regarding differences in demographic and/or clinical characteristics of respondents vs non-respondents

Study design

11. The study sample includes at least 75 patients (arbitrarily chosen)

12. The process of data collection is described

13. The difference in the outcome variable between cancer patients with diabetes and patients with only cancer and/or only diabetes is assessed in multivariable models, including at least 2 covariates

Results

14. Mean, median, standard deviations, or percentages are reported and compared between cancer patients with diabetes and patients with only cancer and/or only diabetes for the most important outcome measures
Number of studies

that scored positive

$N(\%)$

$10(100)$

$10(100)$

$10(100)$

$8(80)$
4 , of which 3 unique, studies had a longitudinal design [19, 20, 24, 25], while the other 6 studies addressed the associations between cancer and diabetes and PROs cross-sectionally [21-23, 26-28]. Most studies focused on patients with specific cancer types including patients with diabetes and prostate [22-25], colorectal [27, 28], or breast cancer [23]. Five studies included cancer patients with diabetes $(\mathrm{CA}+\mathrm{DM}+)$ and made a comparison with cancer patients without diabetes $(\mathrm{CA}+$ DM-) $[21,22,24,25,28]$, one study compared CA+DM+ patients with patients with diabetes only (CA-DM+) [23] and two studies included CA+DM-, CA-DM+ and patients without both diseases (CA-DM-) for comparison [26, 27$]$. Two studies, based on the same data, only included CA+ $\mathrm{DM}+$ and did not include a comparison group [19, 20]. Of the 10 included studies, 8 focused on HRQoL, self-perceived health status, functioning, or symptoms, while 2 studies assessed the impact of cancer and its treatment on diabetes self-management. Most studies used a validated questionnaire. The Short Form (SF)-36 was used most frequently to assess HRQoL or self-perceived health status [21, 22, 25], other studies used the Health Utility Index Mark 3 (HUI3) [26], the EuroQoL Group's EQ-5D [23], the Audit of Diabetes Dependent Quality of Life (ADDQoL) [23], the European Organization for Research and Treatment of Cancer core
Quality of Life Questionnaire (EORTC QLQ-C30) [27], or the University of California, Los Angeles, Prostate Cancer Index (UCLA-PCI) [22, 24, 25]. The EORTC QLQChemotherapy-Induced Peripheral Neuropathy (CIPN)-20 was used to assess neuropathic symptoms [28]. Eight out of 10 studies conducted multivariate analyses and mainly adjusted for socio-demographic [19, 21, 22, 24-28] and cancerrelated covariates $[19,21,22,25,27,28]$, while diabetesrelated covariates [19] and lifestyle factors [24, 26-28] were less often adjusted for.

\section{Study quality}

The 10 included studies scored a mean quality score of 10 out of 14, and scores ranged between 7 and 13. Four studies (40 $\%)$ were classified as being of high quality and 6 (60\%) of adequate quality according to our quality criteria. No studies were considered of low quality. The criteria that were least often met are (\#4) the inclusion of a (healthy) normative sample for comparison, (\#7) a description of at least two clinical variables regarding diabetes diagnosis, and (\#10) information is given regarding differences in demographic and/or clinical characteristics of respondents vs non-respondents (all met by 3 studies) (Table 1). 


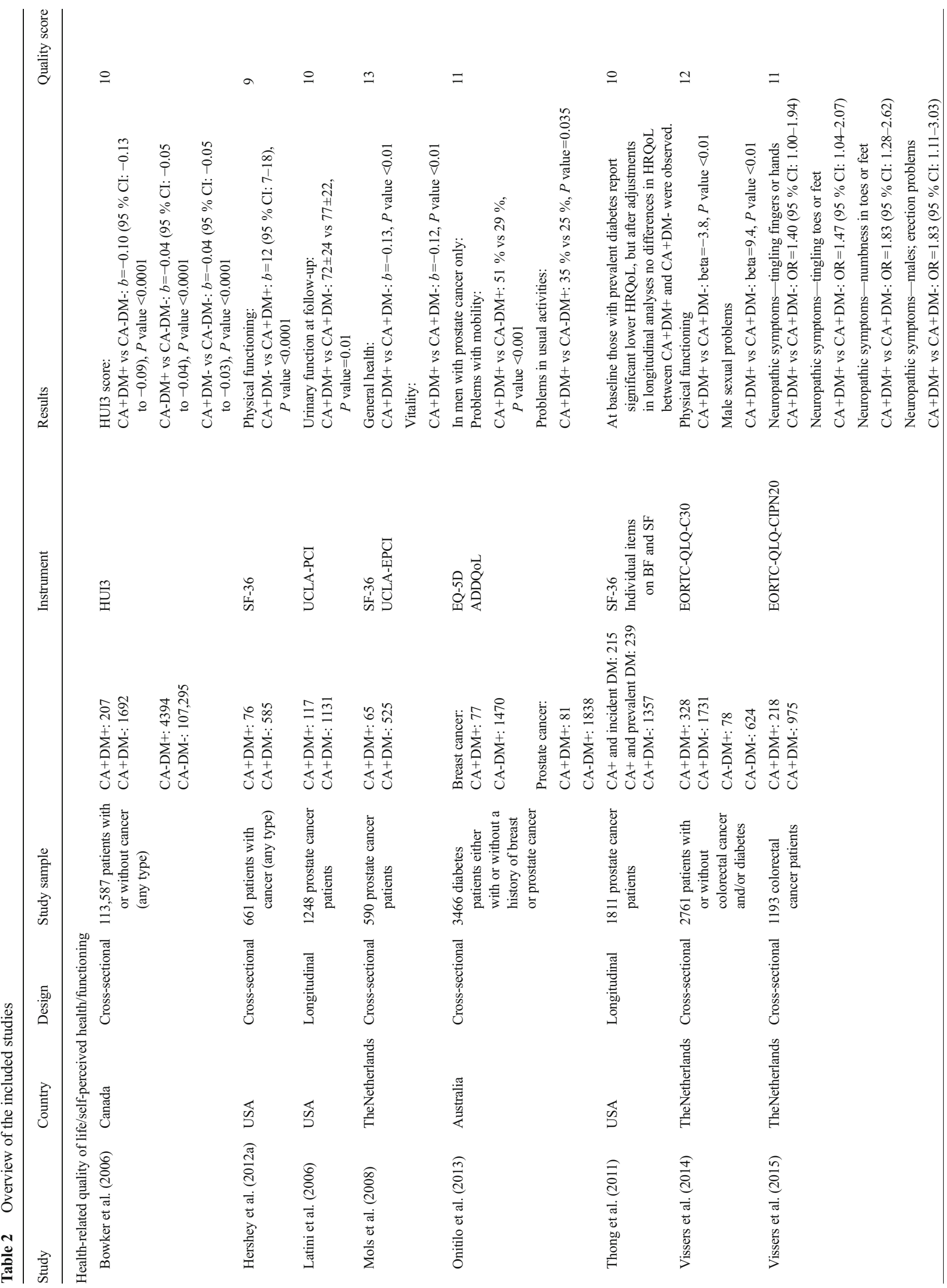




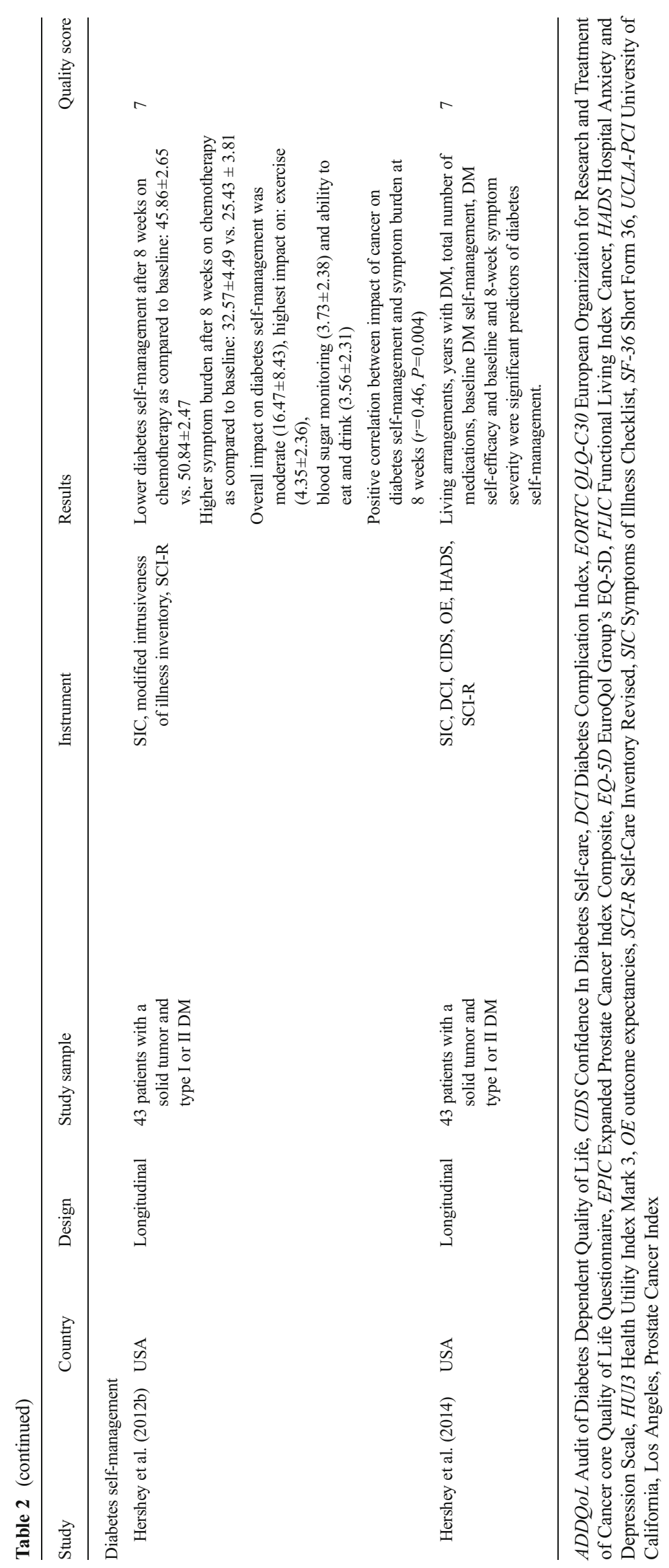


Fig. 1 Flow chart of the selection process of the systematic literature search

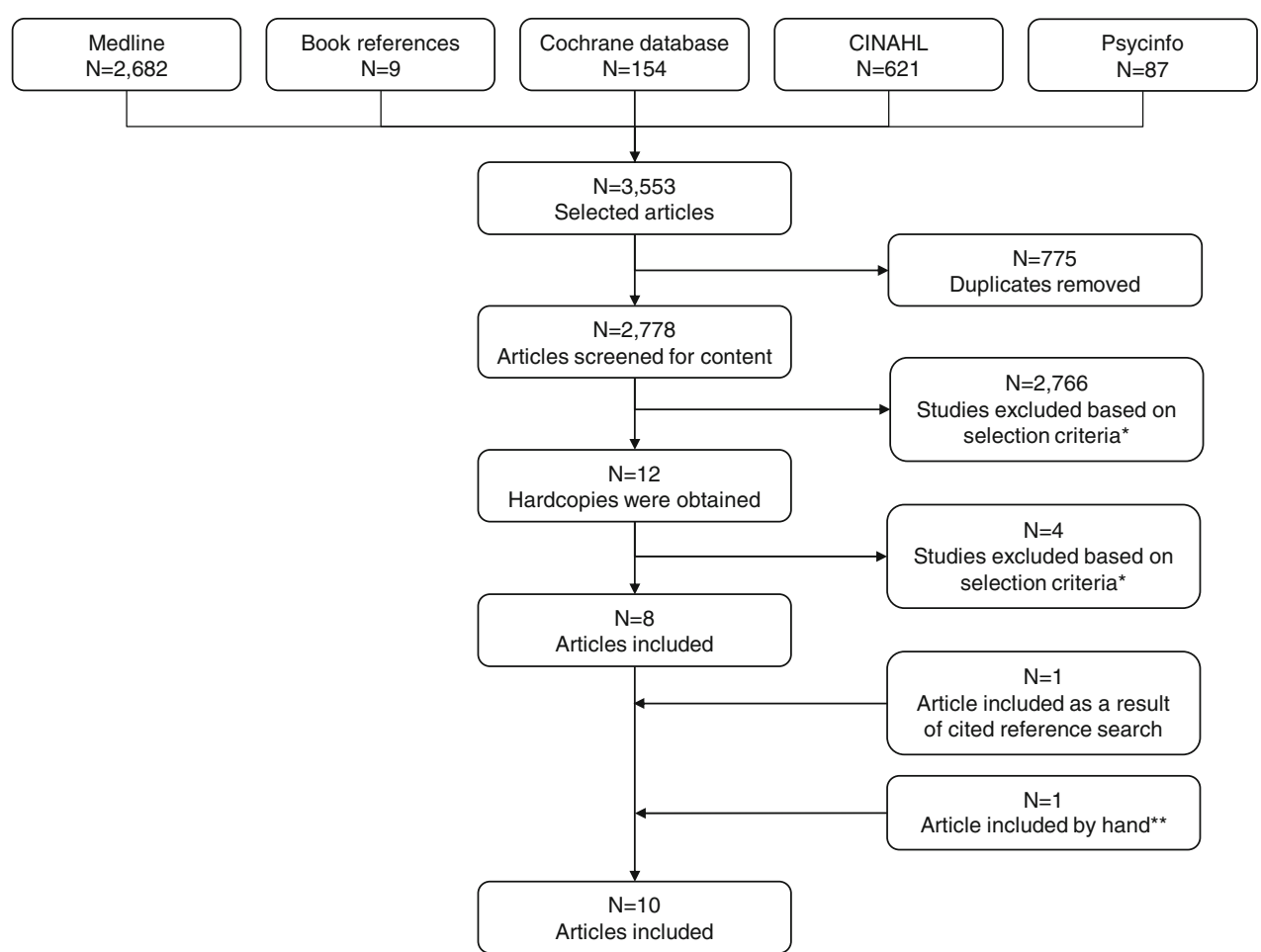

\begin{abstract}
*Selection criteria include: the study (1) includes patients with both cancer and diabetes, (2) PRO is primary or secondary outcome measure of the study, (3) is published in a peer-reviewed journal, and (4) is published in English

** Article of our own research group was accepted for publication on the $13^{\text {th }}$ of January 2015 and published online on 3 February 2015
\end{abstract}

\section{HRQoL, functioning, and symptoms}

All included studies reported worse PROs among $\mathrm{CA}+\mathrm{DM}+$ compared to CA+DM-, CA-DM+, or CA-DM- on at least 1 studied item or subscale, except for 1 longitudinal study [25]. Nine out of the 10 included studies assessed more than 1 PRO, while 1 study only included a general measure of HRQoL [26].

\section{General HRQoL}

A large cross-sectional study conducted in Canada reported lowest average HRQoL scores for CA+DM+ $(n=940)$ followed by CA+DM- $(n=1,692)$, CA-DM+ $(n=4,394)$, and CA-DM- $(n=107,295)$ patients with average HUI3 scores ranging between 0.67 and 0.89 (i.e., where $-0.36=$ worst possible health, $0=$ death, and $1=$ perfect health) [26]. The HUI3 indirectly measures HRQoL using 8 attributes (vision, hearing, speech, ambulation, dexterity, emotion, cognition, and pain) and a mean difference of 0.03 was considered as clinically important. Multivariable regression analyses showed similar results with a lower $\mathrm{HRQ}$ oL for $\mathrm{CA}+\mathrm{DM}+, \mathrm{CA}+$ $\mathrm{DM}-$, and CA-DM+ patients as compared to CA-DM- patients with beta's of $-0.10,-0.04$, and -0.04 , respectively, which was regarded clinically relevant [26]. Similarly, lower general health was reported in a cross-sectional study among 65 prostate cancer patients with vs 525 without diabetes with average SF-36 scores of 51.9 vs 62.5 , which remained significant in multivariable analyses (beta $=-0.13$ ) [22]. A longitudinal study among prostate cancer patients did observe differences between $\mathrm{CA}+\mathrm{DM}+$ and $\mathrm{CA}+\mathrm{DM}$ - in general health at baseline, but after adjustments for age, marital status, educational level, income, employment status, baseline HRQoL, cancer stage, primary treatment, baseline PSA, and baseline Gleason score, this difference did not remain significant [25]. Other studies did not report a worse general health among those with both cancer and diabetes [23, 27].

\section{Physical functioning or mobility}

Five studies included a measure of physical functioning or mobility. In a study with $76 \mathrm{CA}+\mathrm{DM}+$ and $585 \mathrm{CA}+\mathrm{DM}-$, $\mathrm{CA}+\mathrm{DM}+$ scored on average 12 points lower on the physical functioning subscale of the SF-36 as compared to CA+DM[21], as this difference was larger than 0.5 times the standard deviation it can be considered to be clinically relevant [29]. Similarly, a cross-sectional study found more problems with mobility and usual activities among men with prostate $\mathrm{CA}+$ 
$\mathrm{DM}+$ as compared to CA-DM+, but this difference was not found among women with breast cancer [23]. Colorectal CA+ $\mathrm{DM}+$ reported a worse physical functioning as compared to $\mathrm{CA}+\mathrm{DM}-($ beta $=-3.8$ ) [27]. Two studies did not report lower physical functioning among $\mathrm{CA}+\mathrm{DM}+[22,25]$, however, one study did report lower vitality among prostate CA+ $\mathrm{DM}+$ as compared to $\mathrm{CA}+\mathrm{DM}-($ beta $=-0.12)$, which was considered a clinically relevant difference [22].

\section{Sexual functioning}

Sexual functioning was assessed in one study among colorectal $\mathrm{CA}+\mathrm{DM}+[27]$ and in two studies with prostate $\mathrm{CA}+\mathrm{DM}+$ $[24,25]$. Colorectal $\mathrm{CA}+\mathrm{DM}+$ reported more male sexual problems compared to colorectal CA+DM- $($ beta $=9.4)$ in a cross-sectional study from the Netherlands [27]. Among prostate cancer patients, two longitudinal studies did not observe a significant association between comorbid diabetes and sexual functioning $[24,25]$.

\section{Urinary and bowel functioning}

Three studies among prostate cancer $\mathrm{CA}+\mathrm{DM}+$ and $\mathrm{CA}+\mathrm{DM}-$ patients also focused on prostate cancer-specific symptoms, including urinary functioning and/or bowel functioning [22, 24, 25]. One study reported lower urinary function during followup among prostate $\mathrm{CA}+\mathrm{DM}+$ as compared to $\mathrm{CA}+\mathrm{DM}$ (mean score $72 \pm 24$ vs $77 \pm 22$ ) [24], but the other studies did not report differences in urinary or bowel functioning [22, 25].

\section{Neuropathic symptoms}

A cross-sectional study by our research group among 218 colorectal $\mathrm{CA}+\mathrm{DM}+$ and an age- and sex-matched sample of $975 \mathrm{CA}+\mathrm{DM}$ - patients assessed differences in neuropathic symptoms. $\mathrm{CA}+\mathrm{DM}+$ patients reported more neuropathic symptoms regardless of cancer treatment as compared with $\mathrm{CA}+\mathrm{DM}$ - patients regarding tingling fingers or hands $(\mathrm{OR}=$ 1.40; $95 \%$ CI: $1.00-1.94)$, tingling toes or feet $(\mathrm{OR}=1.47$ $95 \%$ CI: 1.04-2.07), numbness in toes or feet $(\mathrm{OR}=1.83$; $95 \%$ CI: 1.28-2.62), and erection problems among men $(\mathrm{OR}=1.83$; $95 \%$ CI: 1.11-3.03) [28]. However, the majority of reported symptoms were of mild severity.

\section{Mental Health}

$\mathrm{CA}+\mathrm{DM}+$ patients did not report worse mental health or emotional functioning compared to CA $+\mathrm{DM}-$ or CA-DM+ in 3 cross-sectional [21, 22, 27] and 1 longitudinal study [25]. One study included a measure of problems with anxiety, but no significant differences were found between prostate or breast $\mathrm{CA}+\mathrm{DM}+$ as compared to $\mathrm{CA}-\mathrm{DM}+$ patients in unadjusted analyses [23].

\section{Diabetes self-management}

Two studies, using the same longitudinal data, addressed problems with diabetes self-management among 43 patients with a solid tumor and type 1 or 2 diabetes [19, 20]. One study showed that patients reported higher scores on symptom burden and lower scores on diabetes self-management after 8 weeks on chemotherapy as compared to baseline (mean $32.57 \pm 4.49$ vs $25.43 \pm 3.81$ and $45.86 \pm 2.65$ vs $50.84 \pm 2.47$, respectively) [20]. In addition, a moderate impact of cancer on diabetes self-management was observed, which mainly affected the ability to exercise, blood sugar monitoring, and ability to eat and drink. Moreover, in qualitative assessments many individuals indicated that they prioritized cancer care instead of diabetes care [20]. The other study mainly focused on predictors of diabetes self-management [19]. This study showed that living arrangements, years with DM, the total number of medications, baseline DM self-management, DM self-efficacy, and baseline and 8-week symptom severity were significant predictors of diabetes self-management, while diabetes complications, cancer type, stage and treatment, outcome expectancies, and anxiety and depression were not [19].

\section{Discussion}

The majority of the included studies in this systematic review (i.e., 8 out of 10 studies) addressed HRQoL, self-perceived health, functioning or symptoms, and two studies, based on the same data, assessed diabetes self-management. In all included studies, $\mathrm{CA}+\mathrm{DM}+$ patients reported worse outcomes, but in 1 longitudinal study among prostate cancer patients, differences disappeared after adjustments [25]. CA+DM+ patients mainly scored lower on general HRQoL [22, 26], physical functioning [21, 23, 27], and sexual functioning [27]. In addition, prostate $\mathrm{CA}+\mathrm{DM}+$ patients reported lower urinary functioning [24] and lower vitality [22], while colorectal CA+ $\mathrm{DM}+$ vs $\mathrm{CA}+\mathrm{DM}$ - patients reported more neuropathic symptoms in a cross-sectional study [28]. Finally, among diabetes patients that also had concurrent cancer, symptom severity increased and diabetes self-management, mainly exercise, blood sugar monitoring, and the ability to eat and drink, was impaired after 8 weeks on chemotherapy [20].

Similar to the results found in our systematic review, literature shows that comorbidity has a significant impact on HRQoL. Several other studies that were not included in this review but included diabetes as one of the studied comorbid conditions showed that cancer patients with comorbidity reported lower HRQoL or functioning [30-33]. A few of those studies reported the impact of diabetes separately and found a poorer general health [30], lower physical functioning [30, 33], more symptoms of nausea [31], and more erection problems among $\mathrm{CA}+\mathrm{DM}+$ men [32]. In line with these results, 
the number of comorbidities, including cancer, among patients with diabetes has also been shown to result in poorer HRQoL [34]. These studies were excluded from the present review as $\mathrm{CA}+\mathrm{DM}+$ patients were not the main sample, and as a result the number of included patients with both diseases was often low.

Although the included studies were of adequate to high quality, they differed substantially in design, population, and methodology. Different instruments were used to measure HRQoL which hampers comparison of the results. Moreover, different cancer types were studied and sample sizes in subgroups were generally low, particularly for $\mathrm{CA}+\mathrm{DM}+$ patients. The majority of studies included $\mathrm{CA}+\mathrm{DM}+$ and $\mathrm{CA}+$ DM- patients, although some studies additionally included a normative sample or CA-DM+ patients for comparison. As a result, information regarding diabetes characteristics was scarce with only 3 out of 10 studies including clinical data regarding diabetes. However, it is important to take the duration and severity of diabetes into account as this may influence the outcomes. Only 4 prospective studies were included, of which 2 were based on the same data, and these studies were conducted mainly among prostate cancer patients.

Despite the heterogeneity in patient samples and PROs studied, this systematic review also has several strengths. It is the first to summarize the literature on PROs among $\mathrm{CA}+\mathrm{DM}+$ patients. In addition, a broad search strategy was used and thereby a complete overview of the previous literature is presented. Finally, the quality of all included studies was assessed by two independent investigators with a 14-item checklist.

\section{Directions for future research}

Although previous studies suggest that having both cancer and diabetes results in worse outcomes, the evidence is scarce and many relevant topics have not been studied yet. This systematic review shows that the majority of studies focused on general HRQoL and physical function, however, only little attention has been paid to mental health. Mental health was assessed in 5 of the 10 included studies but did not appear to be deteriorated in $\mathrm{CA}+\mathrm{DM}+$ patients as compared with $\mathrm{CA}+$ $\mathrm{DM}-$ and CA-DM+ patients. However, this might be a result of the used instrument, as all studies used a subscale of a HRQoL instrument, which might not be sensitive to more specific symptoms of anxiety or depression. Depression is a common problem in both cancer and diabetes patients. Previous research shows that depression is highly prevalent, in about a third of all cancer as well as diabetes patients and is associated with worse prognostic outcomes [35-38]. Therefore, it is possible that $\mathrm{CA}+\mathrm{DM}+$ patients might encounter more mental health issues, which were not picked up in the limited studies in this review. Thus, future studies should focus on mental health issues, including depression among $\mathrm{CA}+\mathrm{DM}+$ patients.
Previous studies show that among both cancer and diabetes patients BMI, physical activity, and smoking are significant predictors of HRQoL [39-43]. However, only 4 of the studies included in this review adjusted for lifestyle factors of which 3 only included BMI $[24,27,28]$ and 1 study additionally adjusted for physical activity and smoking [26]. These studies showed that $\mathrm{CA}+\mathrm{DM}+$ patients have a higher BMI $[24$, 26-28] and are less physically active [26] at baseline than those without diabetes. Although, these studies did observe lower HRQoL among $\mathrm{CA}+\mathrm{DM}+$ vs $\mathrm{CA}+\mathrm{DM}$ - patients independent of the adjustment for lifestyle factors, more research is needed. It is important to assess whether the poorer lifestyle, rather than clinical factors, of $\mathrm{CA}+\mathrm{DM}+$ patients is responsible for the lower HRQoL in this group. Moreover, future research should focus on the effect of changes in lifestyle factors and their impact on HRQoL; with that knowledge, interventions can be developed to improve HRQoL on the long term.

Elderly often live with several chronic illnesses such as cancer and diabetes, which poses a burden on patients. Due to the improved survival, self-management of these chronic diseases is becoming more important. This review included two studies on diabetes self-management which showed that cancer patients performed fewer diabetes self-management behaviors, such as monitoring of the blood glucose levels and exercising, after 8 weeks on chemotherapy [20]. Moreover, qualitative research showed that diabetes patients who develop cancer prioritize their cancer care over their diabetes care [20]. Among diabetes patients, self-management is widely studied and a previous literature review and meta-analysis shows that self-management interventions can improve blood glucose levels, increase knowledge and self-efficacy, and eventually might reduce costs of healthcare utilization [44]. It is important that both patients as well as specialists recognize the importance of self-management of multiple chronic illnesses. It is important that patients are able to utilize their resources and feel that they are in control of life and solve problems when necessary. Therefore, we believe that empowerment of patients and improving self-management behavior are important topics to address in future studies among patients with multiple chronic diseases.

\section{Conclusion}

In conclusion, this systematic review indicates that having both cancer and diabetes results in worse PROs. However, a relatively low number of studies were included and no definitive conclusions can be drawn because of the heterogeneity of the included studies. The included studies were of reasonable quality but a main issue was that clinical information regarding diabetes was missing. More prospective studies with sufficient sample sizes are needed to establish these findings. As this research area is largely neglected and the majority of 
studies focused on HRQoL and physical function, future research should focus on other PROs that are highly prevalent among both cancer and diabetes patients such as mental health, including depression. In addition, as the occurrence of multiple chronic diseases poses important constraints on a person's life and their health care, topics such as self-care and patient empowerment should receive more attention in future research.

Funding The present research was supported by a grant from the European Foundation for the Study of Diabetes (EFSD) to Lonneke V. van de Poll-Franse and Frans Pouwer.

Conflicts of interest The authors declare that there is no conflict of interest associated with this manuscript.

Open Access This article is distributed under the terms of the Creative Commons Attribution 4.0 International License (http:// creativecommons.org/licenses/by/4.0/), which permits unrestricted use, distribution, and reproduction in any medium, provided you give appropriate credit to the original author(s) and the source, provide a link to the Creative Commons license, and indicate if changes were made.

\section{References}

1. de Moor JS, Mariotto AB, Parry C, et al. Cancer survivors in the United States: prevalence across the survivorship trajectory and implications for care. Cancer Epidemiol Biomark Prev. 2013;22: 561-70.

2. Janssen-Heijnen MLG, Houterman S, Lemmens VEPP, et al. Prognostic impact of increasing age and co-morbidity in cancer patients: a population-based approach. Crit Rev Oncol Hematol. 2005;55:231-40.

3. Huxley R, Ansary-Moghaddam A, Berrington de Gonzalez A, et al. Type-II diabetes and pancreatic cancer: a meta-analysis of 36 studies. Br J Cancer. 2005;92:2076-83.

4. El-Serag HB, Hampel H, Javadi F. The association between diabetes and hepatocellular carcinoma: a systematic review of epidemiologic evidence. Clin Gastroenterol Hepatol. 2006;4:369-80.

5. Friberg E, Orsini N, Mantzoros CS, Wolk A. Diabetes mellitus and risk of endometrial cancer: a meta-analysis. Diabetologia. 2007;50: 1365-74.

6. Larsson SC, Mantzoros CS, Wolk A. Diabetes mellitus and risk of breast cancer: a meta-analysis. Int J Cancer. 2007;121:856-62.

7. Larsson SC, Orsini N, Wolk A. Diabetes mellitus and risk of colorectal cancer: a meta-analysis. J Natl Cancer Inst. 2005;97:167987.

8. Larsson SC, Orsini N, Brismar K, Wolk A. Diabetes mellitus and risk of bladder cancer: a meta-analysis. Diabetologia. 2006;49: 2819-23.

9. Kasper JS, Giovannucci E. A meta-analysis of diabetes mellitus and the risk of prostate cancer. Cancer Epidemiol Biomark Prev. 2006; 15:2056-62.

10. van de Poll-Franse LV, Haak HR, Coebergh JWW, et al. Diseasespecific mortality among stage I-III colorectal cancer patients with diabetes: a large population-based analysis. Diabetologia. 2012;55: $2163-72$.
11. Renehan AG, Yeh HC, Johnson JA, et al. Diabetes and cancer (2): evaluating the impact of diabetes on mortality in patients with cancer. Diabetologia. 2012;55:1619-32.

12. Foster C, Wright D, Hill H, et al. Psychosocial implications of living 5 years or more following a cancer diagnosis: a systematic review of the research evidence. Eur J Cancer Care. 2009;18:22347.

13. Stein KD, Syrjala KL, Andrykowski MA. Physical and psychological long-term and late effects of cancer. Cancer. 2008;112:257792.

14. Anderson RJ, Freedland KE, Clouse RE, Lustman PJ. The prevalence of comorbid depression in adults with diabetes: a meta-analysis. Diabetes Care. 2001;24:1069-78.

15. Rubin RR, Peyrot M. Quality of life and diabetes. Diabetes Metab Res Rev. 1999;15:205-18.

16. Bhasin S, Enzlin P, Coviello A, Basson R. Sexual dysfunction in men and women with endocrine disorders. Lancet. 2007;369:597611.

17. Mols F, Vingerhoets AJJM, Coebergh JW, van de Poll-Franse LV. Quality of life among long-term breast cancer survivors: a systematic review. Eur J Cancer. 2005;41:2613-9.

18. Borghouts JAJ, Koes BW, Bouter LM. The clinical course and prognostic factors of non-specific neck pain: a systematic review. Pain. 1998;77:1-13.

19. Hershey DS, Given B, Given C, et al. Predictors of diabetes selfmanagement in older adults receiving chemotherapy. Cancer Nurs. 2014;37:97-105.

20. Hershey DS, Tipton J, Given B, Davis E. Perceived impact of cancer treatment on diabetes self-management. Diabetes Educ. 2012;38:779-90.

21. Hershey DS, Given B, Given C, et al. Diabetes and cancer: impact on health-related quality of life. Oncol Nurs Forum. 2012;39:449 57.

22. Mols F, Aquarius AE, Essink-Bot ML, et al. Does diabetes mellitus as a comorbid condition affect the health-related quality of life in prostate cancer survivors? Results of a population-based observational study. BJU Int. 2008;102:1594-600.

23. Onitilo AA, Donald M, Stankowski RV, et al. Breast and prostate cancer survivors in a diabetic cohort: results from the living with diabetes study. Clin Med Res. 2013;11:210-8.

24. Latini DM, Chan JM, Cowan JE, et al. Health-related quality of life for men with prostate cancer and diabetes: a longitudinal analysis from CaPSURE. Urology. 2006;68:1242-7.

25. Thong MS, van de Poll-Franse LV, Hoffman RM, et al. Diabetes mellitus and health-related quality of life in prostate cancer: 5-year results from the prostate cancer outcomes study. BJU Int. 2011;107: 1223-31.

26. Bowker SL, Pohar SL, Johnson JA. A cross-sectional study of health-related quality of life deficits in individuals with comorbid diabetes and cancer. Health Qual Life Outcomes. 2006;4:17.

27. Vissers PA, Thong MS, Pouwer F, et al. The individual and combined effect of colorectal cancer and diabetes on health-related quality of life and sexual functioning: results from the PROFILES registry. Supportive care in cancer. 2014;22(11):3071-9.

28. Vissers PA, Mols F, Thong MS, et al. The impact of diabetes on neuropathic symptoms and receipt of chemotherapy among colorectal cancer patients: results from the PROFILES registry. J Cancer Surviv. 2015;9(3):523-31.

29. Norman GR, Sloan JA, Wyrwich KW. Interpretation of changes in health-related quality of life: the remarkable universality of half a standard deviation. Med Care. 2003;41:582-92.

30. Dowling EC, Chawla N, Forsythe LP, et al. Lost productivity and burden of illness in cancer survivors with and without other chronic conditions. Cancer. 2013;119:3393-401. 
31. Elliott B, Renier C, Haller I, Elliott T. Health-related quality of life (HRQoL) in patients with cancer and other concurrent illnesses. Qual Life Res. 2004;13:457-62.

32. Pinkawa M, Gagel B, Piroth MD, et al. Erectile dysfunction after external beam radiotherapy for prostate cancer. Eur Urol. 2009;55: 227-36.

33. Vissers PA, Thong MS, Pouwer F, et al. The impact of comorbidity on health-related quality of life among cancer survivors: analyses of data from the PROFILES registry. J Cancer Surviv. 2013;7:602-13.

34. O'Shea MP, Teeling M, Bennett K. Comorbidity, health-related quality of life and self-care in type 2 diabetes: a cross-sectional study in an outpatient population. Ir J Med Sci 2014; 1-8.

35. Spiegel D, Giese-Davis J. Depression and cancer: mechanisms and disease progression. Biol Psychiatry. 2003;54:269-82.

36. Pouwer F, Nefs G, Nouwen A. Adverse effects of depression on glycemic control and health outcomes in people with diabetes: a review. Endocrinol Metab Clin N Am. 2013;42:529-44.

37. Nouwen A, Winkley K, Twisk J, et al. Type 2 diabetes mellitus as a risk factor for the onset of depression: a systematic review and meta-analysis. Diabetologia. 2010;53:2480-6.
38. van Dooren FEP, Nefs G, Schram MT, et al. Depression and risk of mortality in people with diabetes mellitus: a systematic review and meta-analysis. PLoS ONE. 2013;8:e57058.

39. Eckert K. Impact of physical activity and bodyweight on healthrelated quality of life in people with type 2 diabetes. Diabetes Metab Syndr Obes. 2012;5:303-11.

40. Grimmett C, Bridgewater J, Steptoe A, Wardle J. Lifestyle and quality of life in colorectal cancer survivors. Qual Life Res. 2011;20:1237-45.

41. Husson O, Mols F, Ezendam NP, et al. Health-related quality of life is associated with physical activity levels among colorectal cancer survivors: a longitudinal, 3-year study of the PROFILES registry. J Cancer Surviv. 2015;9(3):427-80.

42. Jang S, Prizment A, Haddad T, et al. Smoking and quality of life among female survivors of breast, colorectal and endometrial cancers in a prospective cohort study. J Cancer Surviv. 2011;5:115-22.

43. Li C, Ford ES, Mokdad AH, et al. Clustering of multiple healthy lifestyle habits and health-related quality of life among U.S. adults with diabetes. Diabetes Care. 2007;30:1770-6.

44. Pal K, Eastwood SV, Michie S, et al. Computer-based interventions to improve self-management in adults with type 2 diabetes: a systematic review and meta-analysis. Diabetes Care. 2014;37:1759-66. 